\title{
Neighbor access point identification using station information in High-Density Wi-Fi system
}

\author{
Yuki Katsumata ${ }^{\text {a) }}$, Daisuke Nojima, Takuya Izumisawa, \\ and Akira Yamada \\ Research Laboratories, NTT DOCOMO, INC., \\ 3-6 Hikari-no-oka, Yokosuka-shi, Kanagawa 239-8536, Japan \\ a)yuuki.katsumata.hs@nttdocomo.com
}

\begin{abstract}
In an era where the number of wireless LAN terminals is skyrocketing, ubiquitously improving the quality of the user experience at low cost is paramount. To achieve this, we propose a method that automates re-assignment of frequency channels in high-quality communication areas to achieve automated operation in High-Density Wi-Fi systems. The method identifies neighboring Access Points (APs) to re-assign frequency channels efficiently and effectively. The goal of the proposed method is to clarify the effect of the amount of information including errors on the AP identification accuracy by using a huge amount of station (STA) information. Simulation results indicate that neighboring APs can be identified more accurately using STA information in High-Density Wi-Fi systems.
\end{abstract}

Keywords: neighbor identification, high density, Wi-Fi, WLAN

Classification: Network Management/Operation

\section{References}

[1] L. Wang, C. Hua, R. Zheng, and R. Ni, "Online channel selection and user association in high-density WiFi networks," IEEE International Conference on Communications (ICC), London, UK, pp. 1571-1576, June 2015. DOI:10.1109/ ICC.2015.7248548

[2] M. Riedi, G. G. Basilio, and M. E. Pellenz, "Channel and power allocation algorithm to optimize the performance of large WLANs," ACM Symposium on Applied Computing (SAC), Salamanca, Spain, pp. 673-679, Apr. 2015. DOI:10.1145/2695664.2695718

[3] Y. Han and K. Yang, "Load aware automatic channel switching for softwaredefined enterprise WLANs," KSII Trans. Internet Inf. Syst., vol. 11, no. 11, pp. 5223-5242, Nov. 2017. DOI:10.3837/tiis.2017.11.003

[4] R. Akl and A. Arepally, "Dynamic channel assignment in IEEE 802.11 networks," IEEE International Conference on Portable Information Devices (PORTABLE), Orlando, FL, USA, pp. 1-5, May 2007. DOI:10.1109/ PORTABLE.2007.63

[5] S. Oka, T. Murakami, Y. Takatori, M. Mizoguchi, and F. Maehara, "Performance of adaptive movable access point system in the presence of positioning error," 
IEEE Radio and Wireless Symposium (RWS), Phoenix, AZ, USA, pp. 150-152, Jan. 2017. DOI:10.1109/RWS.2017.7885972

\section{Introduction}

With the proliferation of high-throughput communication services such as livestreaming, broadband services that are accessed by smartphones are required everywhere even in crowded areas. Nevertheless, high-bandwidth communications cannot be provided easily in crowded areas such as sports stadiums due to two major difficulties: (1) Human-body related attenuation of radio signals, and (2) system overload from transmitting and receiving huge amounts of user data and control traffic.

To provide high-bandwidth communications in sports stadiums, we developed a High-Density Wi-Fi system. In these systems, high-performance areas are generated by minimizing the coverage area per Access Point (AP) and increasing the number of APs. In particular, hundreds of APs can be deployed under one Wireless LAN (WLAN) controller, and each AP can accommodate hundreds of stations (STAs).

It is difficult to design a suitable area for each venue for High-Density Wi-Fi systems. The reason for this is that radio propagation characteristics are completely different before and after visitors enter a venue. Venues are rarely filled with visitors without an on-going event and it is difficult to verify the area in advance without any visitors. To design a suitable area, hardware and software measures need to be taken. In terms of hardware, the deployment of APs and antenna orientation must be adjusted. For software, AP and WLAN controller parameters must be adjusted. Since hardware adjustment requires a higher operational cost, software adjustment is more practical. As an example of software adjustment, currently highly-skilled engineers analyze and control a massive number of APs through a WLAN controller. In this paper, we aim to adjust the parameters automatically without intervention by engineers in order to reduce further the operational cost. In particular, we focus on automated re-assignment of frequency channels in HighDensity Wi-Fi systems because frequency channel selection affects the interference between coverage areas and impacts the quality of the user experience.

\section{Automated operations in High-Density Wi-Fi systems}

There are several obstacles to achieving automated operation in High-Density Wi-Fi systems. In this paper, we focus on the issue of frequency channel assignment, which significantly affects performance especially in these systems. In order to maintain communication quality, each AP must transmit radio signals with sufficient strength to satisfy the required Quality of Service (QoS) even at the edge of the cell. Since APs are densely deployed in High-Density Wi-Fi systems, if neighboring APs use the same frequency channel, the QoS in the area supported by the APs significantly degrades due to inter-cell interference. 
In a practical environment, a WLAN controller automatically assigns frequency channels to all APs. However, this assignment does not always work correctly. In such a case, incorrect assignment is detected manually and frequency channels are re-assigned manually. However, manual re-assignment requires continuous monitoring of the network conditions, which is the motivation behind operation automation. For example, Fig. 1 shows frequency channel assignment to 17 APs in a practical venue whose capacity is 1200 people. In Fig. 1a, although there are 19 available channels and there are more channels than APs, the same frequency channel is repeatedly used, e.g. Ch. 36 and Ch. 56. To add to this, the same frequency channel is used in neighboring APs, e.g. Ch. 44. Fig. 1b shows one situation for manual re-assignment.

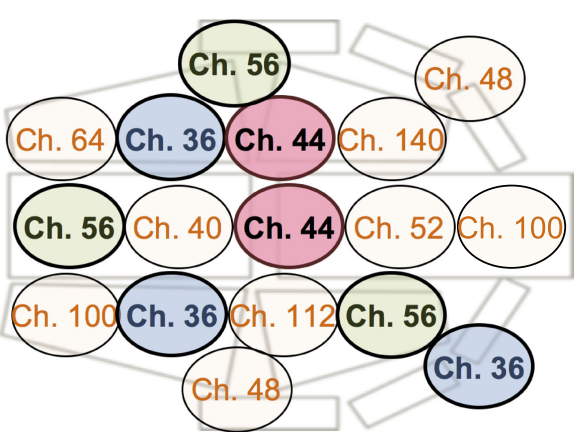

(a) WLAN controller assignment (Does not work correctly consistently)

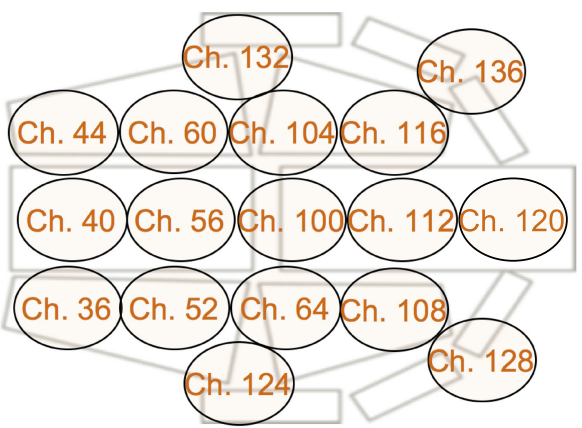

(b) Manual re-assignment by Wi-Fi expert (Works correctly but requires constant monitoring)

Fig. 1. Frequency channels assigned to 17 APs in a practical venue (1200 person capacity).

There are two approaches to address this issue. The first one is to improve the assignment algorithm. In this research area, several techniques have been proposed. For example, by using airtime cost (routing metric in IEEE802.11s) [1], the received signal strength indicator (RSSI) [2], or the AP traffic load [3], the respective authors attempted to estimate the appropriate AP frequency channel.

The second approach is to re-assign frequency channels based on manual reassignment. Toward this end, we focus on identification of neighboring APs because the interference from neighbors using the same frequency channel have the largest impact on performance. If we can identify neighboring APs, we can use the existing assignment method in [4].

The latter approach yields two advantages. One is that we can apply assignment know-how obtained through previous manual re-assignment. The other is that we can implement the approach at low cost because we do not need to change the implementation of existing Wi-Fi equipment.

We aim to identify neighboring APs automatically only using information obtained from a WLAN controller. Although we can use the AP deployment map, the map does not include precise information pertaining to barriers such as floors, obstacles, and their construction materials. Such information depends on the venue and obtaining it requires additional cost. Therefore, it is necessary to identify 
neighboring APs without any input from outside the Wi-Fi network. In [5], the authors estimated the appropriate positions of APs using the RSSI and time of arrival.

The characteristic of the proposed method is to identify neighboring APs using AP and STA information. This information includes the RSSI or the signal-to-noise ratio collected via a WLAN controller. Many existing studies have shown that since these network observation values contain many errors due to noise, these values are not sufficient to estimate positions with sufficient accuracy. Certainly, it is difficult to estimate an AP position or even neighboring APs using only AP information. However, if a large amount of STA information is used, the accuracy can be improved.

The point of the proposed method is to clarify the effect that the amount of information has on the identification accuracy of neighboring APs. Two factors affect the identification accuracy. First, when the amount of information becomes larger, the errors become averaged. This should improve the accuracy. Second, when the amount of information becomes larger, more errors are accumulated. This should worsen the accuracy. Thus, the purpose of this study is to confirm that the large amount of information obtained in High-Density Wi-Fi systems improves the identification accuracy.

\section{Identification of neighboring APs}

The proposed method comprises three steps as shown in Fig. 2.
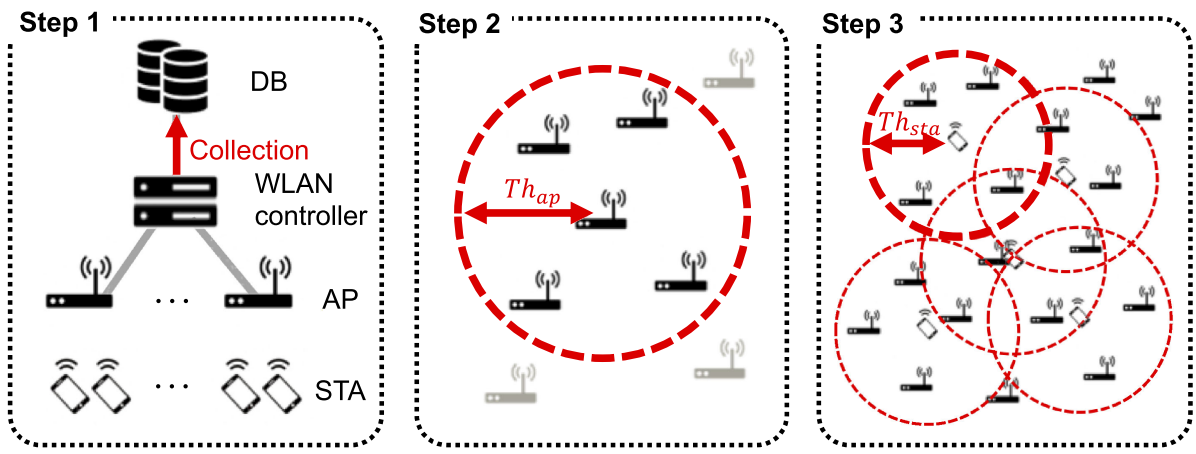

Fig. 2. Concept for proposed method.

Step 1. Information collection from WLAN controller. An external database gathers AP and STA information via the WLAN controller. This information includes the AP RSSI from other APs and the STA RSSI from APs. The database accumulates multiple sampled information sets at a specific time point.

Step 2. Identification of neighboring APs using AP information. A threshold is used to identify if an AP is neighboring another AP. We define some variables: $R S S I_{i, j}$ is the RSSI of $\mathrm{AP} i$ from $\mathrm{AP} j ; T h_{a p}$ is the RSSI threshold; and $N_{i, j}$ represents whether or not AP $i$ neighbors AP $j$. Here, $N_{i, j}=1$ indicates that AP $i$ neighbors AP $j$. Then, this step is given as 


$$
N_{i, j}= \begin{cases}1 & \left(R S S I_{i, j}>T h_{a p}\right) \\ 0 & \left(R S S I_{i, j} \leq T h_{a p}\right)\end{cases}
$$

Step 3. Identification of neighboring APs using STA information. A threshold is used to identify if a STA is nearby an AP. Then, based on the obtained results, another threshold is used to identify if an AP is nearby another AP. This step is based on the assumption that two APs are neighbors if both APs are nearby a STA. We define some variables: $R S S I_{i, x}$ is the RSSI of AP $i$ from STA $x$ and $T h_{s t a}$ is the RSSI threshold. Note that $T h_{s t a}$ is different from $T h_{a p}$ because the STA transmission power is different from that of the APs and the RSSI is also different. Term $N_{i, x}$ represents whether or not AP $i$ neighbors STA $x$. Here, $N_{i, x}=1$ indicates that AP $i$ neighbors STA $x$ and $T h_{c n t}$ is the threshold for the number of neighboring STAs for an AP. Then, this step is given as

$$
\begin{gathered}
N_{i, x}= \begin{cases}1 & \left(R S S I_{i, x}>T h_{s t a}\right) \\
0 & \left(R S S I_{i, x} \leq T h_{s t a}\right)\end{cases} \\
N_{i, j}=\left\{\begin{array}{cl}
1 \quad\left(\sum_{\forall x \in S T A} N_{i, x}>T h_{c n t} \cap \sum_{\forall x \in S T A} N_{j, x}>T h_{c n t}\right) \\
0\left(\sum_{\forall x \in S T A} N_{i, x} \leq T h_{c n t} \cup \sum_{\forall x \in S T A} N_{j, x} \leq T h_{c n t}\right)
\end{array}\right.
\end{gathered}
$$

\section{Simulation evaluation}

We evaluate the identification accuracy of the neighboring APs using only AP and STA information. For ease, a method employing Steps 1 and 2 (AP information only) is compared to that using Steps 1 and 3 (STA information).

We define the simulation environment hereafter. Standard IEEE 802.11ac is used. The radio frequency is U-NII-1 and U-NII-2C. The number of APs is 19. The AP cell radius is $0.01 \mathrm{~km}$. The number of STAs is 1900 . The AP location is indicated in Fig. 3a. STAs are located randomly in the field. We assume that AP $i$ neighbors AP $j$ if the side of the AP $i$ cell is shared with the $\operatorname{AP} j$ cell as shown in Fig. 3a. For example, the APs that neighbor AP ${ }^{\#} 1$ are AP ${ }^{{ }^{*}} 2,{ }^{\#} 3, \ldots,{ }^{\#} 7$. The

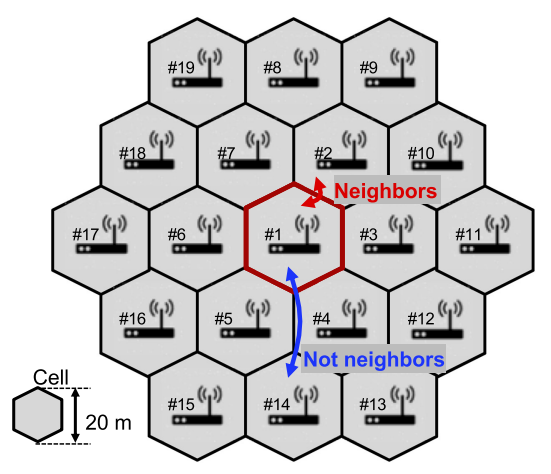

(a) AP location and cell in simulation

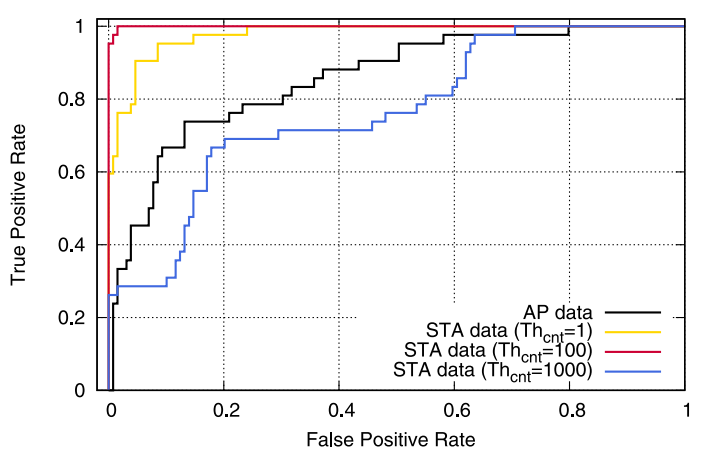

(b) Simulation results

Fig. 3. Simulation evaluation. 
parameters are $T h_{a p}$ for Step 2 and $T h_{s t a}$ for Step 3. We compare the obtained results using the proposed methods by adjusting parameters with the neighbors assumption.

The results are shown in Fig. 3b. The horizontal axis shows the false positive rate and the vertical axis shows the true positive rate. The results are shown as receiver operating characteristic curves. The black, yellow, red, and blue curves respectively represent the identification accuracy using only AP information, using STA information when $T h_{c n t}$ is 1 , using STA information when $T h_{c n t}$ is 100 , and using STA information when $T h_{c n t}$ is 1000 . The results show that identification using STA information tends to be more accurate than when using AP information. Parameter $T h_{c n t}$ represents the number of votes for the majority. Thus, so long as $T h_{c n t}$ is not an excessively large value, e.g., $T h_{c n t}$ is 1000 for 1900 STAs (blue curve in Fig. 3b), the large amount of STA information improves the identification accuracy for neighboring APs. In other words, the results indicate that the neighboring APs are identified accurately by using STA information in HighDensity Wi-Fi systems.

\section{Conclusion}

In this paper, our aim was to actualize automated re-assignment of frequency channels as a way to achieve operation automation in High-Density Wi-Fi systems. In order to actualize efficient and effective re-assignment, the identification of neighboring APs is necessary. Toward this end, we proposed an identification method using a large amount of STA information. Simulation results indicated that the neighboring APs can be identified more accurately using STA information than by using only AP information in High-Density Wi-Fi systems. 